\section{AL-AZHAR}

Assiut Dental Journal
The Official Publication of The

Faculty of Dental medicine.

Al-Azhar Assiut Uniuersity.

Egypt

\title{
Occlusal Splint with Arthrocentesis as Combination Therapy for Treatment of TMJ Internal Derangement
}

\author{
Ahmed M. Rashid*, Bahaa El- Din A. Tawfek
}

Codex : 08/2020/04

Aadj@azhar.edu.eg

\section{KEYWORDS}

TMJ, Occlusal Splint,

Arthrocentesis,

Internal Derangement,

Limited Mouth Opening

1. Department of Oral and Maxillofacial surgery, Faculty of Dental Medicine (Boys),Cairo, Al-Azhar University, Egypt.

* Corresponding Author e-mail: ahmedrashed.9@azhar.edu.eg

\begin{abstract}
Aim: This study was designed to evaluate the occlusal splint with arthrocentesis as combination therapy for treatment of TMJ internal derangement. Subjects and methods: Forty-two patients (ranging in age 23-54 years) suffering from signs of TMJ internal derangement attending at Oral and Maxillofacial Surgery Department at Sayed Galal hospitals (Al Azhar university).The patients complaints were limited mouth opening,TMJ pain, and joint noises during function. The patients will be divided randomly into three groups .Group I: Patients in this group will be treated by occlusal splint .Group II : Patients in this group will be treated by arthrocentesis (saline injection) .Group III: Patients in this group will be treated by combination of arthrocentesis and occlusal splint .Each group contained patients suffering from anterior disc displacement with and without reduction. Clinical evaluation was performed before the procedure and at 1,2 weeks,1,2,3, and 6 months postoperatively .Maximum mouth opening,pain with visual analogue scale, and joint sound also were recorded at each follow-up visit. Results: All groups of this study showed improvement in maximal mouth opening,TMJ pain, and clicking sounds with variable degrees according to treatment modality. Conclusion: Although in patients with TMJ ID benefitted from each technique to a variable extent, arthrocentesis of TMJ followed by splint seemed to be superior in achieving rapid and prolonged improvement in TMJ ID compared with other techniques.
\end{abstract}

\section{INTRODUCTION}

Temporomandibular joint dysfunction (TMD) is a clinically significant condition which can be a source of acute or chronic orofacial pain and dysfunction including limitation of mandibular movement ${ }^{(1)}$. This condition may cause pain, clicking, crepitus and irregular or deviating jaw function. From a clinical point of view, TMJ internal derangement has two expressions: painful clicking and chronic closed lock which is associated with osteoarthritis ${ }^{(2)}$.Cardinal signs of TMJ disorder's (internal derangement) may be (a) limitation of mandibular movement (b) pain with mandibular function (c) joint sounds ${ }^{(3)}$. 
The primary goal in the treatment of TMD is to reduce pain or mandibular dysfunction ${ }^{(4)}$. TMD treatment can be divided into two categories: conservative method and surgical method. Conservative treatments include medication, habit modification, counseling, physical therapy, splint therapy, and manipulation ${ }^{(5)}$. Surgical treatments include arthrocentesis, arthroscopy, arthroplasty, discectomy, and TMJ reconstruction ${ }^{(6)}$. A conservative, reversible method is widely accepted to be the first choice for TMJ treatment ${ }^{(7)}$. As one of the conservative treatments, occlusal splint has been frequently used for internal derangement and myofascial pain treatment ${ }^{(8)}$.

Occlusal adjustment involves repositioning the mandible to a centric position by using prosthodontic or orthodontic appliances. Intraoral occlusal splints are designed to provide even and balanced occlusal contact without forcefully altering the mandibular rest position or permanently altering the dental occlusion. Occlusal splint made of processed hard acrylic, a splint is worn on the teeth like retainer or a removable denture ${ }^{(9)}$.

This splint is often successful, but the length of time required to reach a pain-free normal range of motion is sub-optimum. Among the surgical interventions, arthrocentesis is generally suggested for patients who are not responsive to conservative therapy ${ }^{(8)}$. However arthrocentesis is effective for washing out bradykinin, interleukin- 6 and protein from the TMJ ${ }^{(1)}$.

The aim of arthrocentesis carried out in degenerative joint diseases is to remove the inflamed synovial fluid from the joint space, provide appropriate synovial fluid viscosity, and remove the adhesions by the application of hydraulic pressure (10). This study sought to test the hypothesis that the simultaneous use of occlusal splint and arthrocentesis is an effective method for TMJ internal derangement .

\section{AIM OF THE STUDY}

The aim of the present study was to evaluate the occlusal splint with arthrocentesis as combination therapy for treatment of TMJ internal derangement.

\section{PATIENT AND METHODS}

The study were included forty-two patients (ranging in age 23-54 years) suffering from signs of TMJ internal derangement attending at Oral and Maxillofacial Surgery Department at Sayed Galal hospitals (Al Azhar university).

The patients complaints were limited mouth opening,TMJ pain, and joint noises during function.

\section{Grouping of patients:}

The patients will be divided randomly into three groups.

Group I: Patients in this group will be treated by occlusal splint .

Group II : Patients in this group will be treated by arthrocentesis (saline injection) .

Group III: Patients in this group will be treated by combination of arthrocentesis and occlusal splint.

\section{Selection criteria:}

\section{- Inclusion criteria:}

- Male and female patients with internal derangement of the TMJ

- Diagnostic criteria of internal derangement included a history of TMJ noise, anterior disk displacement, limitation of mandibular movement, pain with mandibular function and joint sounds.

\section{- Exclusion criteria:}

- Patients with systemic disease such as rheumatoid arthritis.

- Degenerative change of condylar head. 
- $\quad$ Trauma at TMJ.

- Patients with the anomalies of the face and jaw.

- Patients with history of primary surgical treatment in TMJ.

\section{a- Clinical evaluation:}

The patient was seated in anatomical position "head supported" in order

To avoid any Movements from cervical vertebra, then ask the patient for:

1. Maximum mouth opening: to measure the distance from the incisal edge of the maxillary central incisor to the incisal edge of the mandibular central incisor on a digital caliper .

2. TMJ palpation: for detection of any tender area at the joint by applying finger tips over TMJ on both sides.

3. TMJ sounds: using a stethoscope over the TMJ and ask the patient to open to the maximum then to close, doing lateral movement and protrusion retrusion movement.

4. Muscle palpation: detection if there is any pain or tenderness on temporails,masseter,internal \& external pterygoid, trapezius and sternocleidomastoid mucles.

5. After all patients had been examined, Pain was measured with a visual analogue scale (VAS, $10-\mathrm{cm}$ line), which ranged from 0 (no pain) to 10 (worst possible pain).

Clinical examination was performed before treatment and at 1,2 weeks, 1,2,3,and 6 months after treatment.

\section{b- Imaging evaluation:}

- Magnetic resonance imaging (MRI) were taken to detect disc position and abnormalities of TMJ. MRI was obtained to confirm the initial diagnosis of anterior disc displacement (fig1).
- A surface coil $(7.5 \mathrm{~cm})$ was used to examine the TMJ bilaterally at the same time.Images were taked before treatment and 6 monthes after treatment

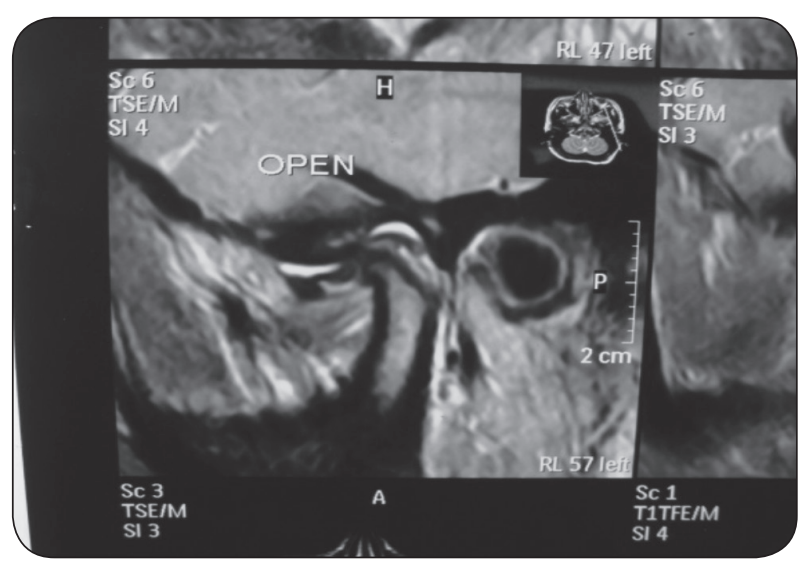

Fig. (1) MRI showing anterior disc displacement without reduction in open position.

\section{Splint fabrication (fig 2)}

An alginate impression of the maxillary arch was performed by which a master cast was fabricated for each patient.

Full coverage hard acrylic stabilizing splint, $3 \mathrm{~mm}$ thinckness on the maxilla.

The splint was retaianed by adams clasp on the upper first molars .

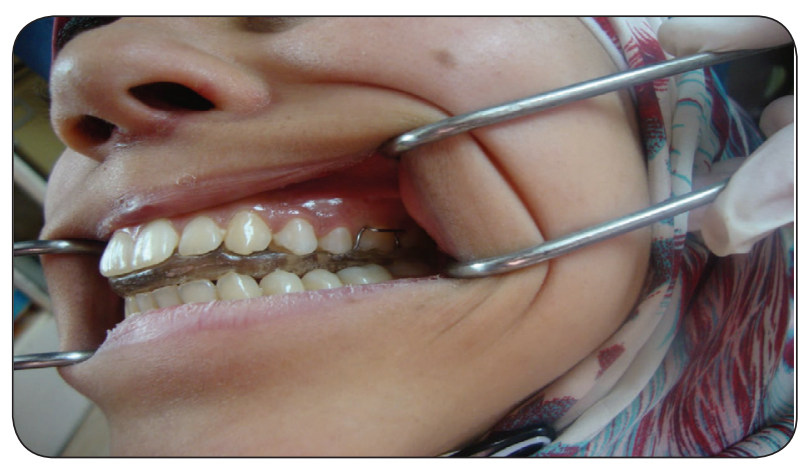

Fig. (2) Occlusal splint

The splint was worn for 6 months in the following manner: The 1 st week, patients were instructed to wear the splinit for only 2 hours at the first day, then, increasing the period of wearing the splint 
gradually by more 2 hours daily to reach 14 hours/ day at the end of the 1 st week. At the 2 nd week: splint was worn constantly for 14 hours daily . at 3rd week, patients were instructed to increase the period of wearing the splint again gradually by more 2 hours daily to reach 24 hours/day except meal time and the patient was instructed to wear the device till the end of 6th month.

\section{Lavage technique:}

- The operation started by blocking the auriculotemporal nerve (ATN) with mepivacaine HCL 2\% (with levonordefrin 1:20000) (*) followed by posterior deep temporal and masseter nerves anesthesia with one or two carpules to prevent discomfort and/or pressure pain which may occur at the beginning of the joint lavage procedure.

- This provides optimal region analgesia, preventing the need for sedation.

- The target site was prepared, isolated by sterile drapes and scrubbed surface with betadine .

- The points of needle insertion was marked on the skin as a line was drawn from the middle of the tragus to the outer canthus and entry points marked along this canthotragal line(HolmlundHellsing Line). 1st point (posterior entrance point) which corresponds to the glenoid fossa was marked $10 \mathrm{~mm}$ from the midtragus and 2 $\mathrm{mm}$ below the line which represent injected point and 2nd point (anterior entrance point) which corresponds to the articular eminence also corresponds to out flow of saline will marked $10 \mathrm{~mm}$ from the first point and $10 \mathrm{~mm}$ below the line.

- Then a 18 gauge needle was introduced at $1 \mathrm{st}$ point and 2-3 $\mathrm{ml}$ of saline was injected through this needle to distend the joint space.Another 18 gauge needle was then inserted at the 2 nd point to establish a free flow of the solution through

(*)(produced by pharmaceuticals Alexandria Egypt) the joint space.A $20 \mathrm{ml}$ syringe filled with saline was injected into the superior joint space through the 1 st needle and 2 nd needle provides an outflow for saline. A total of 100-200 ml solution was used to lavage the superior joint space.(fig 3)

- During this procedure the mandible was manipulated in the vertical,protrusive, and lateral directions to free up the disc.

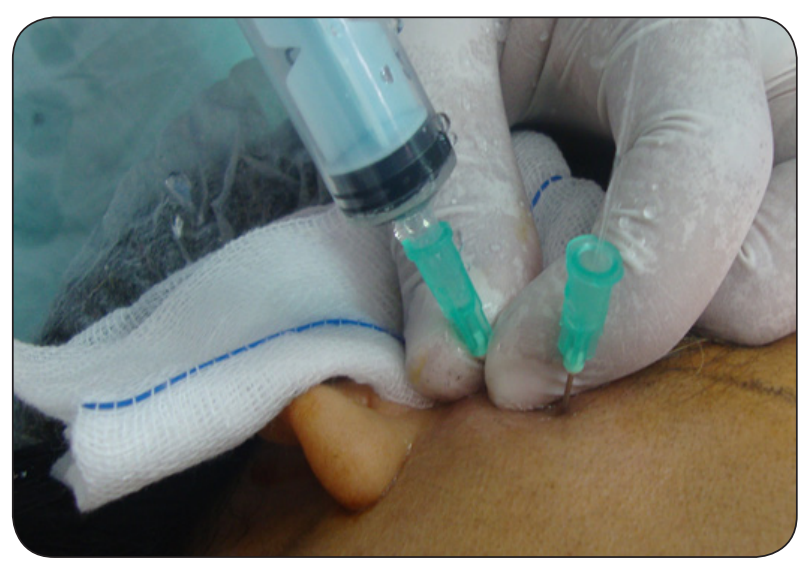

Fig. (3) Photograph showing intra- articular injection of saline

\section{Post-operative care:(for group 2.3)}

1. Patients were instructed to apply cold fomentation over the surgical site area for 15 minutes each hour the day of surgery to decrease the amount of edema postoperatively

2. Hot fomentation over the surgical site area on the day after to improve the blood circulation and reduce inflammation

3. All patients were given an antibiotics Emox $500 \mathrm{Mg}($ ) 4 time per day as a prophylactic measure to overcome any possibility of joint space infection and Flagyl 500mg tab ( ) three times daily were advised for aweek.

NSAIDS: Brufen 600mg tab( )two times daily for aweek.

Anti edematous drug: Alphentern tab ( ) three times daily were advised for 1 week. 
4. Moreover the patients were instructed to receive a soft diet in order to decrease the effort over the joint, further more do active and passive mouth opening exercises.

5. Finally the patients instructed to wear the occlusal splint for six month after the procedure (for group 3).

\section{RESULTS}

A total of 42 patients with painful TMJ (Internal darangement ) were enrolled in this study. Out of these, 14 patients treated with occlusal splint, 14 patients treated with arthrocentisis and 14 patients treated with(splint+arthrocentisis).The patients were followed for six months.

After arthrocentisis pain was expressed immediately and for 24 hours after . with moderate to sever then gradually decrese after medication and application of cold ' hot fomentation over site of injection which completely dissappared in the 3 rd to 7 th day after procedure. 3 parameters were evaluated in this study visual anloge scale VAS, maximum mouth opening MMO and joint sound.

\section{1- TMJ pain.}

In comparing pain in all groups there was gradual reduction in the mean of values of pain scores recorded on VAS during the 6th monthes follow up period started from the 1 st week after the proce- dures then every week in the 1st month followed by monthely followed up showing more reduction in pain score until the end of six months. Reduction in pain was more in (Group 3) than (Groups 1,2)

\section{2- Maximum mouth opening.}

Fig. 4 summarize the assessment of MMO at the study intervals .Occlusal splint group(group1) showed significant increase in the MMO after 3 and 6 months postoperatively while after 1,2 weeks and 1,2 months there was no significant difference.

In the Arthrocentesis group(group2), there is statistically insignificant increase in the MMO after 1 week postoperatively followed by significant increase after 2 weeks then icrease monthely till 6 months.

In combination group ( group 3) there is no significant difference between preoperatively and (1week postoperatively ) while significant difference between preoperatively and (2week, 1, 2, 3 , and 6 months postoperatively ). Comparisons between the 3 groups showed statistically insignificant difference between them preoperatively and 1 week postoperatively, after 2 weeks, 1,2,3 and 6 months the mean MMO values in group3 are statistically significantly higher than group1 and group2.

\section{3- Clicking.}

Improved across treatment in all groups espacialy in group 3.

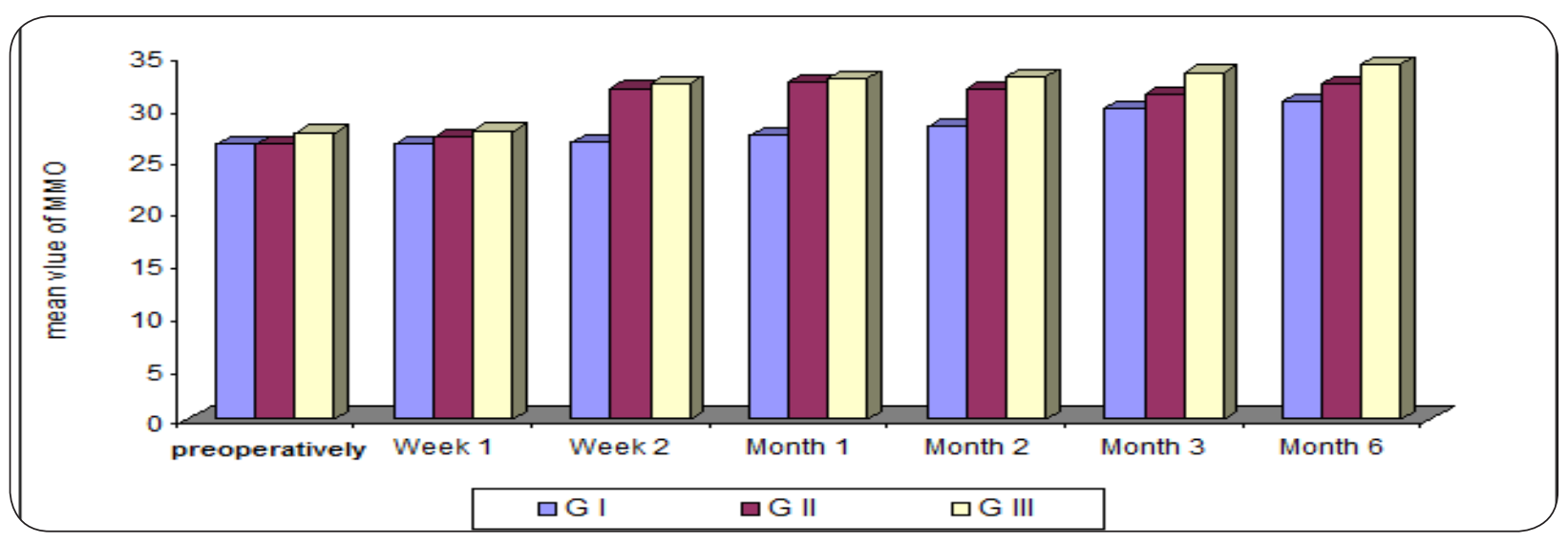

Fig. (4) Abar chart showing mean values of mouth opening through out the study period. 
The MRI showed that the disc did not reduce to its normal physiologic relation with the condyle and articular eminence in all groups .There was marked reduction in joint effusion and decrease in signal intensity of retrodiscal tissue(becoming pseudodisc)

\section{DISCUSSION}

Temporomandibular disorders (TMDs) are considered the major cause of orofacial pain. Displacement of the articular disc can result in decreased joint space, joint noise (clicking, popping, or crepitation), arthritis, condylar resorption, inflammation, and compression of the bilaminar tissue, all of which can cause various degrees of pain and dysfunction. ${ }^{(1)}$ The present study compared the effectiveness of arthrocentesis, splint treatment, and a combination of both therapies. A total of 42 patients with painful TMJ (Internal derangement) were enrolled in this study. 14 patients were treated with Occlusal splint(group1), 14 patients were treated with arthrocentisis (group2) and14 patients were treated with (splint + arthrocentisis (group3). The patient was followed for six months.

The needles were used in this study instead of canuala,which is more difficult to enter the narrow joint spaces, according to Nitzan et al., 1991, ${ }^{(12)}$ using single needle is indicated for hypomobile joints, with strong adhesions, or for joints with degenerative changes which make difficult insertion of the second needle. ${ }^{(12)}$ Double needle technique was used in this study because it is more efficient in washing out of the joint.

In this study using of $100 \mathrm{~mm}$ of saline was sufficient to clear the joint space of blood, loose particles, inflammatory mediators and pain mediators .In addition, it is possible to lyse any possible adhesions and to release the joint disc by hydraulic pressure .This is in agreement with Nitzan et al., $1991^{(12)}$. Zardeneta et al., 1997, ${ }^{(13)}$ who reported that infusions of $100 \mathrm{~mL}$ are sufficient for an effective therapeutic washing.
All patients subjectively had moderate to severe pain preoperatively. There was significant difference in pain between the groups. Reduction in pain was more in (Group 3) than (Groups 1,2), patients reported a significant reduction in TMJ pain after six monthes in all groups, but the combination treatment was superior to arthrocentesis alone or splint alone due to rapid improvement by arthrocentesis, the pain reduction is attributed to the high pressure irrigation which washes away inflammatory mediators and providing pain relief, and continuous improvement by wearing splint which lead to reduction of the excessive loading on the joint, relax the masticatory muscles, and support the adaptation of the articular structures, and this goes in agreement with Machon et $\mathrm{al}^{(14)}$ who showed that arthrocentesis combined with splint therapy achieved higher success rates than splint treatment alone.

In this study, Mouth opening was observed to be increased across the follow up period in all groups; however, the mouth opening was significantly higher in (Group 3) than in (Group 1,2). This attribuated to that arthrocentesis under sufficient pressure can also remove adhesions, widen the joint spaces and improve mouth opening. Similar results were achived by Guarda-Nardini et $\mathrm{al}^{(15)}$ by using the same arthocentesis technique and their results showed improvement in mouth opening due to over expansion of the capsule and breaking the adherence that were responsible for reduce the translator movement through arthrocentesis, and splint led to breake in pain-spasm-pain cycle that lead to decrease in muscle spasm and increase in MMO. This finding is consistent with the findings of Park et al. ${ }^{(16)}$ showing that the combination of arthrocentesis and stabilization splint for TMD Patients enabled an increase in the amount of maximum mouth opening and decrease in the average value of pain.

In terms of clinically detectable clicking sound, there was significant improvement in clicking in group (3) more than group (1) due to increase in intra articular space (disc room increased) by occlusal splint and adhesions release that lead to widening of the joint spaces by arthrocentesis. 
These findings are consistent with Kovaleski et al (17) who have also shown a significant reduction in clicking of TMJ, tenderness of TMJ and muscles in response to occlusal splint therapy when patient were followed up for 2 monthes. On the other hand in group 2, Joint sound in the current study showed no significant improvement in all the treatment period which agreed with (Ohnuki et al) ${ }^{(5)}$ who reported that arthrocentesis do not necessarily improve the position and deformity of the disc . Also Alpaslan C and Alpaslan GH ${ }^{(18)}$ reported no significant decrease in clicking sound in patients receiving arthrocentesis only.

As a result, all groups in this study showed improvement in pain, MMO, and noise. For MMO, there was a significant difference by treatment. Group 2 took longer time to achieve normal state than group 3, while group 1 took longer time than group 2. For both pain and MMO, a statistically significant difference was noted. Group 3 took less time to achieve normal state than group 2 and group 1. A more effective result was observed in group 3 than in the two other groups at the initial stage.

Finally it is important to begin with the release of inflammatory mediators of the joint disc by arthrocentesis and proper lavage of the joint space, and in the case of success, to maintain this state by applying a repositioning occlusal splint.

Patients submitted only for arthrocentesis had enjoyed pain relief for a period with return of symptoms at the end of the study. This can be explained by the fact that arthrocentesis can eliminate joint effusion causing pain, and improves the pain condition of the joint but cannot maintain permanent reduction of load on intra capsular tissues as can splint. In this study MRI was done for all patient at the end of the follow up period .However, no changes appeared in MRI in relation to the preoperative one, which may be attributed to the short term follow up period .Similary previous studies with same findings claimed that there is no correlation between the clinical outcomes and MRI findings in short term studies. $\left.{ }^{(19-20}\right)$

\section{CONCLUSIONS}

1. Occlusal splint remain the gold conservative treatment for TMJ internal derangements.

2. Lavage of superior joint space with saline exerts its effects through its ability to eliminate joint effusion thus reducing the symptoms. These observations make arthrocentesis proposed line of treatment option for patients with TMJ internal derangements.

3. Arthrocentesis of TMJ followed by splint seemed to be superior in achieving rapid and prolonged improvement in TMJ ID compared with other techniques.

\section{REFERENCES}

1. Al-Said S., Abdullah N. and Ragab H. Comparative study of arthrocentesis with or without using piroxicam in the management of temporomandibular joint disorders. Alex dent j. 2015; 40.160-65.

2. Wadhwa S, Kapila S. Tmj disorders: future innovations in diagnostics and therapeutics. J dent educ. 2008;72: 930-47.

3. Peagle D, Holmund A, Hjerpe A. Expression of peotoglycan mrna in patients with painful clinking and chronic closed lock of tmj. Int j oral maxillofac surg. 2005; 34: 656-58.

4. Karlis V, Glickman R. Nonsurgical management of temporomandibular disorders. In: Miloro M, Ghali G, Larsen P, Waite P. Peterson's principles of oral and maxillofacial surgery. 2nd ed. Hamilton: bc decker. 2004:949-50.

5. Ohnuki T, Fukuda M, Nakata A, Nagai H, Takahashi T, Sasano T. Evaluation of the position, mobility, and morphology of the disc by mri before and after four different treatments for temporomandibular joint disorders. Dentomaxillofac radiol.2006; 35:103-09.

6. Choi J, Kim I, Oh N, Oh S, Kim E, Lee S. A clinical study in the prognosis of the temporomandibular disorder. $\mathrm{J}$ korean assoc oral maxillofac surg. 2000;26:497-506.

7. Hupp J, Ellis E, Tucker M. Contemporary Oral and Maxillofacial Surgery. $5^{\text {th }}$ ed. St. Louis: Mosby. 2008:617-650.

8. Lee H., Baek H., Song D, Kim H., Kim H., Kim B. et al. Effect of simultaneous therapy of arthrocentesis and occlusal splints on temporomandibular disorders: anterior disc displacement without reduction. J Korean Assoc Oral Maxillofac Surg. 2013; 39: 14-20. 
9. Seifeldin S. and Elhayes K. Soft versus hard occlusal splint therapy in the management of temporomandibular disorders (tmds). Saudi Dent J. 2015; 27: 208-14.

10. Önder M, Tüz H, Koçyiğit D. and Kişnişci R. Long-term results of arthrocentesis in degenerative temporomandibular disorders. Oral Surg Oral Med Oral Pathol Oral Radiol Endod. 2009; 107: 1-5.

11. Schiffman E, Ohrbach R, Truelove E, Look J, Anderson G, Goulet JP, et al. Diagnostic Criteria for Temporomandibular Disorders (DC/TMD) for Clinical and Research Applications: Recommendations of the International RDC/ TMD Consortium Network* and Orofacial Pain Special Interest Group $\dagger$. J Oral Facial Pain Headache 2014;28:6-27.

12. Nitzan D, Dolwick M, Martinez G . Temporomandibular joint arthrocentesis: a simplified treatment for severe, limited mouth opening . Journal of oral and maxillofacial surgery: official jounal of the American Association of Oral and Maxillofacial Surgeons. 1991;49:1163-7.

13. Zardeneta G, Milam SB, Schmitz JP. Elution of proteins by continuous temporomandibular joint arthrocentesis. J Oral Maxillofac Surg 1997;55:709-16.

14. Machon V, Hirjak D, Lukas J. Therapy of the osteoarthritis of the temporomandibular joint. J Craniomaxillofac Surg 2011;39: 127-30.

15. Guarda-Nardini L, Manfredini D, Ferronato G. Arthocentesis of the temporomandibular joint: a proposal for a single-needle technique. Oral Surgery, Oral Medicine,
Oral Pathology, Oral Radiology, and Endodontology. 2008;106:483-6.

16. Park YH, Lee SH, Yoon HJ. An effect of combination with arthrocentesis and stabilization splint treatment on temporomandibular joint disorder patient. J Korean Assoc Maxillofac Plast Reconstr Surg 2010;32:32-6.

17. Kovaleski WC, Beaver De. J: Influence of occlusal splints on jaw position and musculature in patients with temporomandibular joint dysfunction. J Prosthet Dent 1975; $33: 321-7$

18. Alpaslan C, Bilgihan A, Alpaslan GH, Güner B, Ozgu“ r Yis M, Erbas, D, Effect of arthrocentesis and sodium hyaluronate injection on nitrite, nitrate, and thiobarbiturate acid-reactive substance levels in the synovial fluid. 2000;89:686-90.

19. Lee SH, Yoon HJ.MRI findings of patients with tempromandibular joint internal derangement: before and after performance of arthrocentesis and stabilization splint. Journal of oral and maxillofacial surgery: official journal of American Association of Oral and Maxillofacial Sourgens. 2009;67:314-7.

20. Emshoof R, Innerhofer K, Rudisch A, Betram S. Clinical versus magnetic resonance imaging finding with internal derangement of the tempromandibular joint:an evaluation of anterior disc displacement without reduction. Journal of oral and maxillofacial surgery:official journal of the American Association of Oral and Maxillofacial Surgeons.2002:60:36-41. 


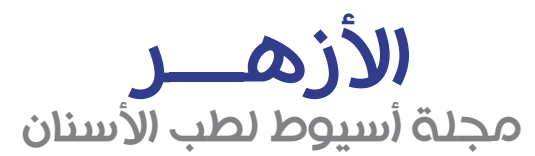

النشر الرسمي لكلية طب الأسنان جامعة الأزهر أسيوط الكاية

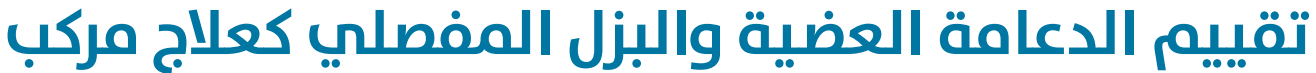 لعلاج الاضطراب الداخلي لمفصل الفك الصدغي كلئ}

\section{احمد محمد راشد *، بهاء الدين عبدربـه توفيق}

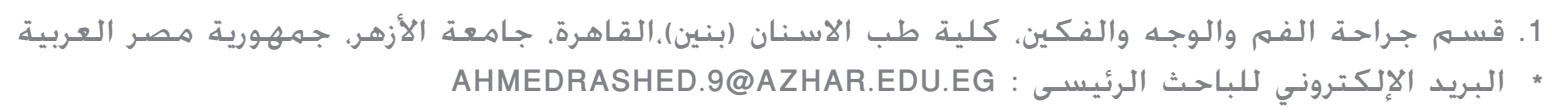

(الملخص:

الهدف: تم تصميم هذه الدراسة لتقييم الدعامة العضية والبزل المفصلي كعلاج مركب لعلاج الاضطراب الداخلي لمفصل الفك الصدغي

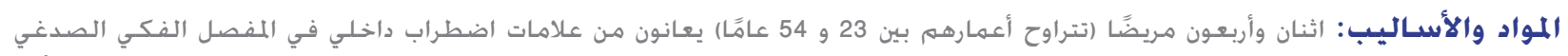

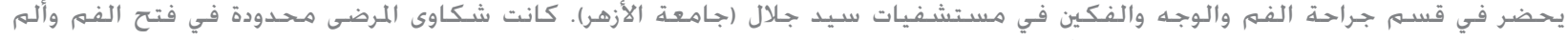

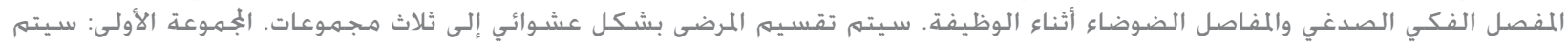

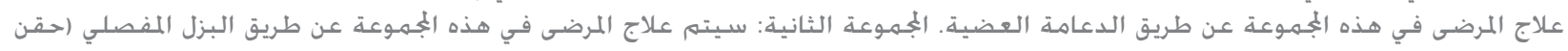

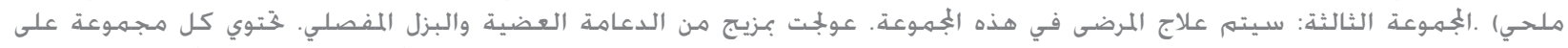

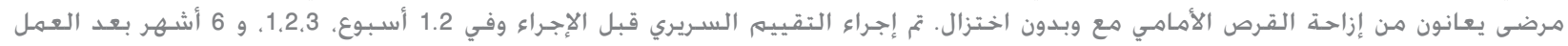

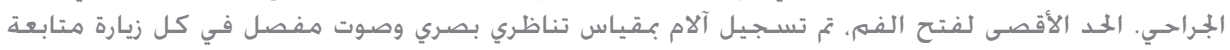

النتائج: والأصوات الناجتة من مفصل الفك الصدغي أظهرت جهيع مجهوعات هذه الدراسة ثنسنا في فتح الفم الأقصى، والألم بدرجات متغيرة وفقا لطريقة العلاج.

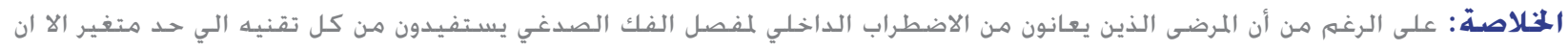

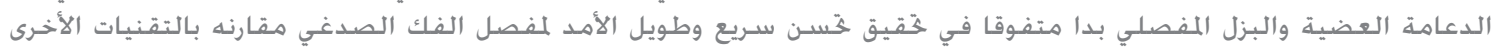
الكلمات المفتاحية: مفصل الفك الصدغي .الدعامة العضية، البزل المفصلي ، لاضطراب الداخلي لمفصل الفك الصدغي. المد الأقصى لفتح الفم الفمات 